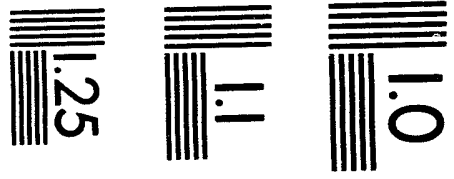

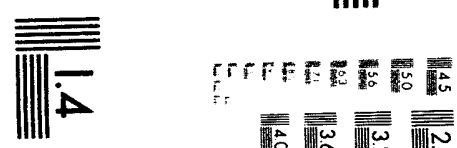

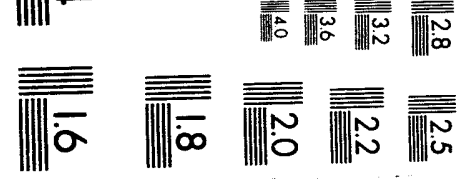



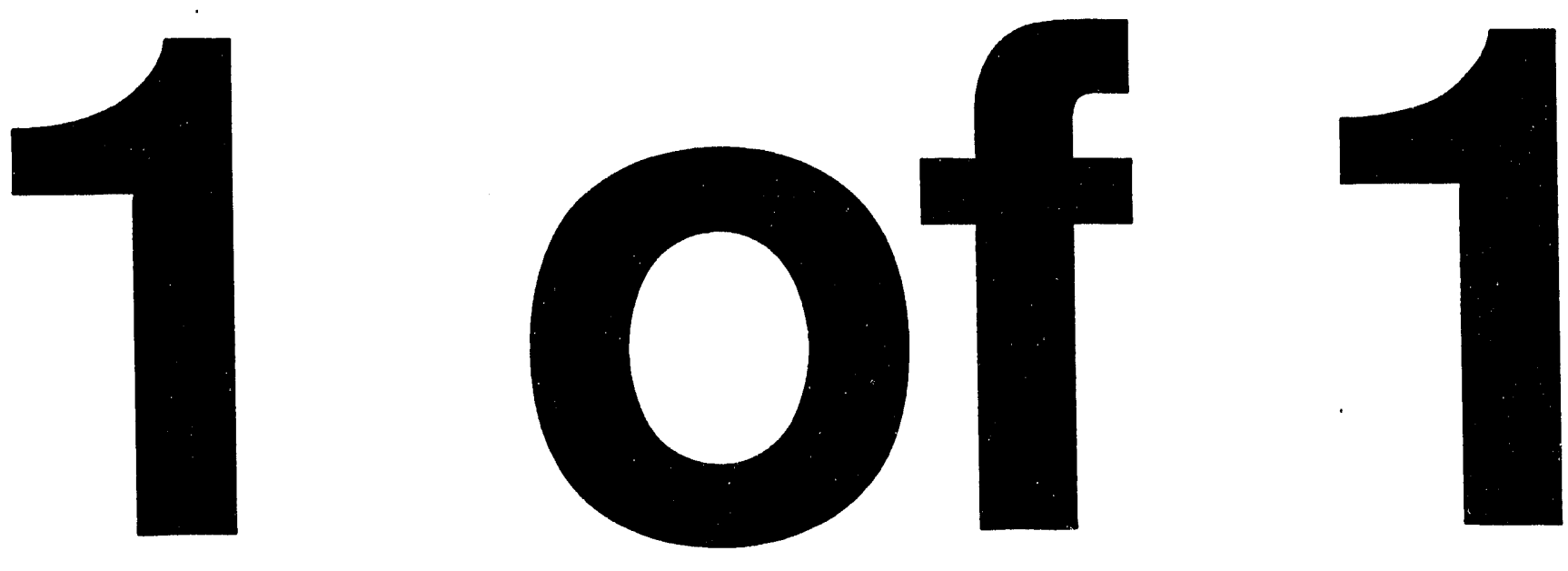
FIRST PRINCIPLES PSEUDOPOTENTIAL CALCULATIONS

\title{
ON ALUMINUM AND ALUMINUM ALLOYS
}

\author{
J.W. Davenport, ${ }^{1}$ N. Chetty, ${ }^{1}$ R.B. Marr, ${ }^{2}$ S. Narasimhan, ${ }^{1}$ \\ J.E. Pasciak, ${ }^{2}$ R.F. Peierls, ${ }^{2}$ and M. Weinert ${ }^{1}$ \\ Brookhaven National Laboratory* \\ Department of Physics ${ }^{1}$ \\ Department of Applied Sciences ${ }^{2}$ \\ Upton, New York 11973
}

\begin{abstract}
Recent advances in computational techniques have led to the possibility of performing first principles calculations of the energetics of alloy formation on systems involving several hundred atoms. This includes impurity concentrations in the $1 \%$ range as well as realistic models of disordered materials (including liquids), vacancies, and grain boundaries. The new techniques involve the use of soft, fully nonlocal pseudopotentials. iterative diagonalization, and parallel computing algorithms. This approach has been pioneered by Car and Parrinello. Here we give a review of recent results using parallel and serial algorithms by our group on metallic systems including liquid aluminum and liquid sodium, and also new results on vacancies in aluminum and on aluminummagnesium alloys.
\end{abstract}

\section{DISCLAIMER}

This report was prepared as an account of work sponsored by an agency of the United States Government. Neither the United States Government nor any agency thereof, nor any of their employees, makes any warranty, express or implied, or assumes any legal liability or responsibility for the accuracy, completeness, or usefulness of any information, apparatus, product, or process disclosed, or represents that its use would not infringe privately owned rights. Reference herein to any specific commercial product, process, or service by trade name, trademark, manufacturer, or otherwise does not necessarily constitute or imply its endorsement, recommendation, or favoring by the United States Government or any agency thereof. The views and opinions of authors expressed herein do not necessarily state or reflect those of the United States Government or any agency thereof.

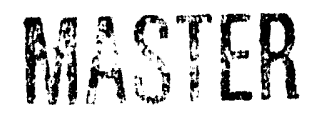




\section{Introduction}

Recent advances in pseudopotential electronic structure calculations, pioneered by Car and Parrinello [1,2], have led to the possibility of studying alloys with relatively large crystallographic unit cells. In addition to the complex alloy phases often seen in real systems, this includes models of impurities, grain boundaries, dislocations, and even liquids using a supercell approach. These techniques lend themselves naturally to parallel computing and as a result are excellent candidates for early utilization of parallel machines. As an example, we point to the aluminum-magnesium system. Alloys with a few percent magnesium have found application in the ubiquitous soda can [3] as well as in many other areas $[4,5]$. Yet the $A l-M g$ system does not contain any simple crystalline compounds [6]. Instead it is dominated by two complex phases, $\alpha\left(A l_{12} M g_{17}\right)$ which has the $\alpha$ manganese structure with 29 atoms per unit cell and $\beta\left(A l_{3} M g_{2}\right)$ with a complex face centered cubic (fcc) structure containing roughly 300 atoms. Thus, aluminum alloys seem to be interesting model systems for the study of alloy formation in complex phases.

The purpose of this paper is to describe briefly these new techniques, to present results for several simple structures of aluminum-magnesium, and to describe a parallel implementation which has been applied to the problem of vacancies in crystalline aluminum.

\section{Techniques}

The calculations described here all use the plane wave pseudopotential method to solve the Schrodinger equation self consistently and compute the charge density and total energy using the local density approximation (LDA). An excellent review of this technique has been given by Pickett [7]. The advantages of this method are that the codes are relatively straightforward - hence lending themselves to easy transportability to new machines. Also they make no shape approximations to the charge density or potential, and the force acting on a given atom and the stresses are easily and accurately calculated. A disadvantage is that large numbers of plane waves are required especially for large unit cell systems and for transition metals, oxides, and carbides. However, as originally pointed out by Car and Parrinello, large basis sets can be handled because only about $1 \%$ of the eigenvalues need to be computed. In addition, progress has been made in reducing the size of the basis for transition metals by a new generation of soft pseudopotentials $[8,9]$.

Since the pseudopotential local density method is well known $[2,7]$, we only mention here the points which are essential for treating large systems.

The wave function is expanded in a plane wave basis

$$
\psi(\vec{r})=\sum_{m} \exp \left\{i\left(\vec{k}+\vec{g}_{m}\right) \cdot \vec{r}\right\} C_{m}
$$

where $m$ labels the reciprocal lattice vectors and $\vec{k}$ lies within the first Brillouin zone. The local density approximation (LDA) then requires diagonalization of a Hamiltonian matrix

$$
h_{m m^{\prime}}=\frac{1}{2}\left|\vec{g}_{m}\right|^{2} \delta_{m m^{\prime}}+U^{\text {local }}\left(\vec{g}_{m}-\vec{g}_{m^{\prime}}\right)+U_{m m^{\prime}}^{\text {nonlocal }} \text {. }
$$

The local part of the potential contains the Coulomb repulsion of the other electrons, the exchange-correlation potential, and the local part of the pseudopotential. The nonlocal 
part is usually given in the Kleinman-Bylander form [10]

$$
U_{m m^{\prime}}^{\text {nonlocal }}=\sum_{\lambda \mu} A_{m j \lambda \mu}^{*} A_{m^{\prime} j \lambda \mu}
$$

Here $j$ labels the atoms in the unit cell and $(\lambda \mu)$ refers to the angular momentum component of the nonlocal pseudopotential.

For free electron metals such as magnesium and aluminum one needs of order 100 plane waves per atom to achieve good convergence. Therefore, a 100 atom supercell would lead to a Hamiltonian matrix of $10,000 \times 10,000$ which can not be handled by current computers with conventional diagonalization techniques.

On the other hand, only a small fraction, of order $1 \%$, of the eigenvectors are required. For example, $A l$ has 3 electrons per atom and accounting for spin 150 states would be occupied in a 100 atom supercell. Typically, for metals, extra states are included and occupied with a Fermi distribution [11].

This naturally leads to the use of iterative diagonalization techniques. The simplest of these is the power method [12]. We form a sequence

$$
\psi^{n+1}=h \psi^{n}
$$

It is easy to show that for an arbitary input vector $\psi^{\circ}$, repeating this process and renormalizing at each step leads to the eigenfunction $\psi$ which has the largest eigenvalue. Clearly, the closer $\psi^{\circ}$ to the actual eigenfunction the more rapid the convergence. For example, in the Car-Parrinello molecular dynamics method [1], the ions move a short distance in each time step, typically $\sim 0.01 \AA$, and therefore the eigenvector from the last time step is an excellent input for the current one.

We also note that it is not necessary to store the full matrix $h$, but only the eigenvectors $\psi^{n}$. Since these are of order $1 \%$ of the total, the savings in storage is a factor of 100 .

Car and Parrinelio suggested an algorithm for molecular dynamics simulations which they derived in an elegant fashion from a fictitious Lagrangian $[1,2]$

$$
\psi^{n+1}=2 \psi^{n}-\psi^{n-1}+\delta^{2} / \mu(\lambda-h) \psi^{n} .
$$

Here $\delta$ is the time step of the molecular dynamics simulation, $\mu$ is the "fictitious" mass and $\lambda$ the current estimate of the eigenvalue

$$
\lambda=\left\langle\psi^{n}|h| \psi^{n}\right\rangle .
$$

This algorithm has been applied to many systems including liquid and amorphous silicon [13], liquid sodium [14], and liquid aluminum [15].

Our algorithm [16] is similar to that of Car and Parrinello, and to the preconditioned conjugate method [2]

$$
\psi^{n+1}=\psi^{n}+\left(h_{d}+s\right)^{-1}(\lambda-h) \psi^{n} .
$$

Here $h_{d}$ is the diagonal part of $h$, shifted by $s$ which guarantees all eigenvalues are positive definite. We follow this by a subspace diagonalization where the subspace consists of the eigenfunctions included in the Fermi distribution. 
Table 1: Lattice Constants for $M g$ and $A l$

\begin{tabular}{|c|c|c|c|}
\hline & $a$ & $c$ & $c / a$ \\
\hline$\underline{M g \text { (hcp) }}$ & & & \\
Theory & $3.04 \AA$ & $5.20 \AA$ & 1.71 \\
Expt & $3.21 \AA$ & $5.21 \AA$ & 1.62 \\
\hline Al (fcc) & & & \\
Theory & $3.96 \AA$ & & 1.71 \\
Expt & $4.05 \AA$ & & \\
\hline
\end{tabular}

The important features of all these schemes are that one need not store the full $h$ matrix and that the product $h \psi$ can be evaluated with fast Fourier transform techniques in $G \log _{2} G$ operations rather than $G^{2}$ ( $G$ is the dimension of the $h$ matrix). It is also crucial to use the Kleinman Bylander form, Eq. (3), for the nonlocal pseudopotential.

This nonlocal part scales as the number of atoms to the third power, as does the subspace diagonalization, and this will ultimately limit the size of system which can be studied. We believe this will nevertheless enable calculations on systems containing $\sim 1000$ atoms.

\section{Aluminum Magnesium}

As a first example we report results for the lattice constants and heats of formation of several simple $A l-M g$ compounds. The $A l$ and $M g$ pseudopotentials were generated by the method of Bachelet Hamann and Schluter [17] but without using their analytic fits. We first calculated the lattice constants for aluminum in the fcc structure and magnesium in the hcp structure. The results are shown in Table 1 and are in essential agreement with previous calculations $[18,19]$. It is typical of the local density approximation that it underestimates the lattice constants by several percent.

We next computed the lattice constants and energies of the compounds $\mathrm{Al}_{3} \mathrm{Mg}$, and $\mathrm{AlMg}_{3}$ in the $\mathrm{Ll}_{2}\left(\mathrm{Cu}_{3} \mathrm{Au}\right)$ structure and $\mathrm{AlMg}$ in the $\mathrm{Ll} l_{0}(\mathrm{CuAuI})$ structure.

Figure 1 shows the energy versus lattice constant for $A l M g$ at various values of $c / a$. The energy is minimized by $c / a=1$ and $a=4.14 \AA$. These calculations require only a few minutes on a Cray Y-MP C90 and are an illustration of the relative ease with which geometries can be determined.

Figure 2 shows the deviations from Vegard's law in these compounds: The upper curve uses the calculated volume per atom of $A l$ and $M g$. The experimental point is for the compound $A l_{12} M g_{17}$. The lower curve uses the experimental volume per atom of $A l$ and $M g$. The points at $x=0$ and $x=1$ represent the error in the theoretical volume for the pure constituents.

Finally, in Figure 3 we show the heat of formation of these compounds. It is defined in the usual way as the energy per atom of the compound minus the concentration weighted average of the energy of the constituents. That is

$$
\Delta H=E_{A l_{(1-X)} M g_{x}} / N-(1-X) E_{A l}-X E_{M g}
$$




\section{$E_{\text {tot }}$ vs. a for AlMg in CuAu Structure}

(4 atoms per unit cell)

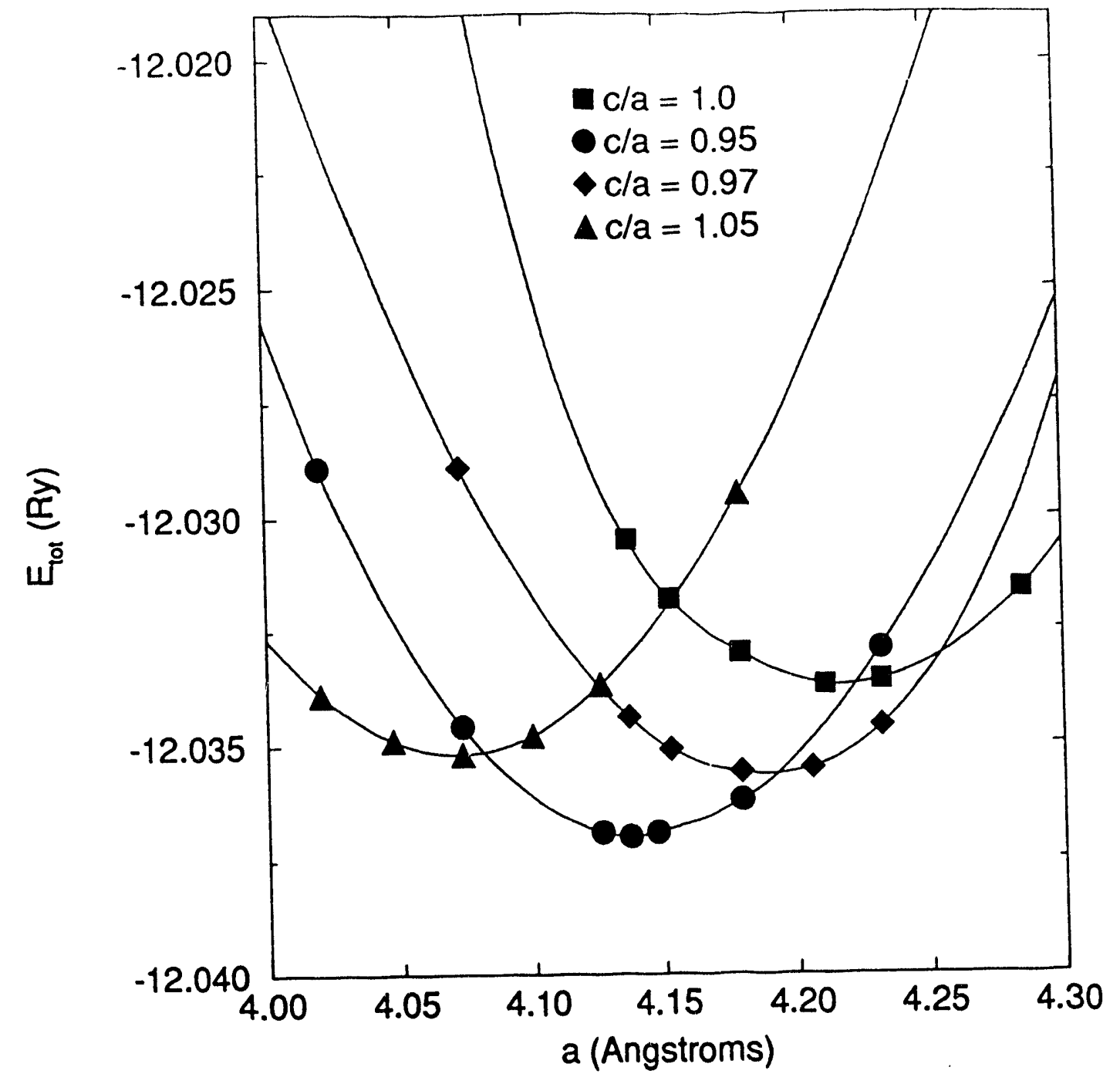

Figure 1: Energy versus Lattice Constant for $A l M g$ in the $L l_{0}(C u A u I)$ Structure for Various Values of $c / a$.

where $N$ is the number of atoms per unit cell. The experimental value lies significantly below the calculated ones which is consistent with the fact that none of these simple phases exist.

\section{Parallel Computing}

The iterative diagonalization schemes described in Eqs. (5-7) lend themselves naturally to the new generation of parallel machines. We have adopted the strategy of performing all of the operations connected with the "update" in Eq. (7) on a single processor. In the case that there are enough processors to handle all the states, then they can all be updated simultaneously. If not, they can easily be done in batches. Since each update 


\section{Deviations from Vegard's Law for AIMg Alloys}

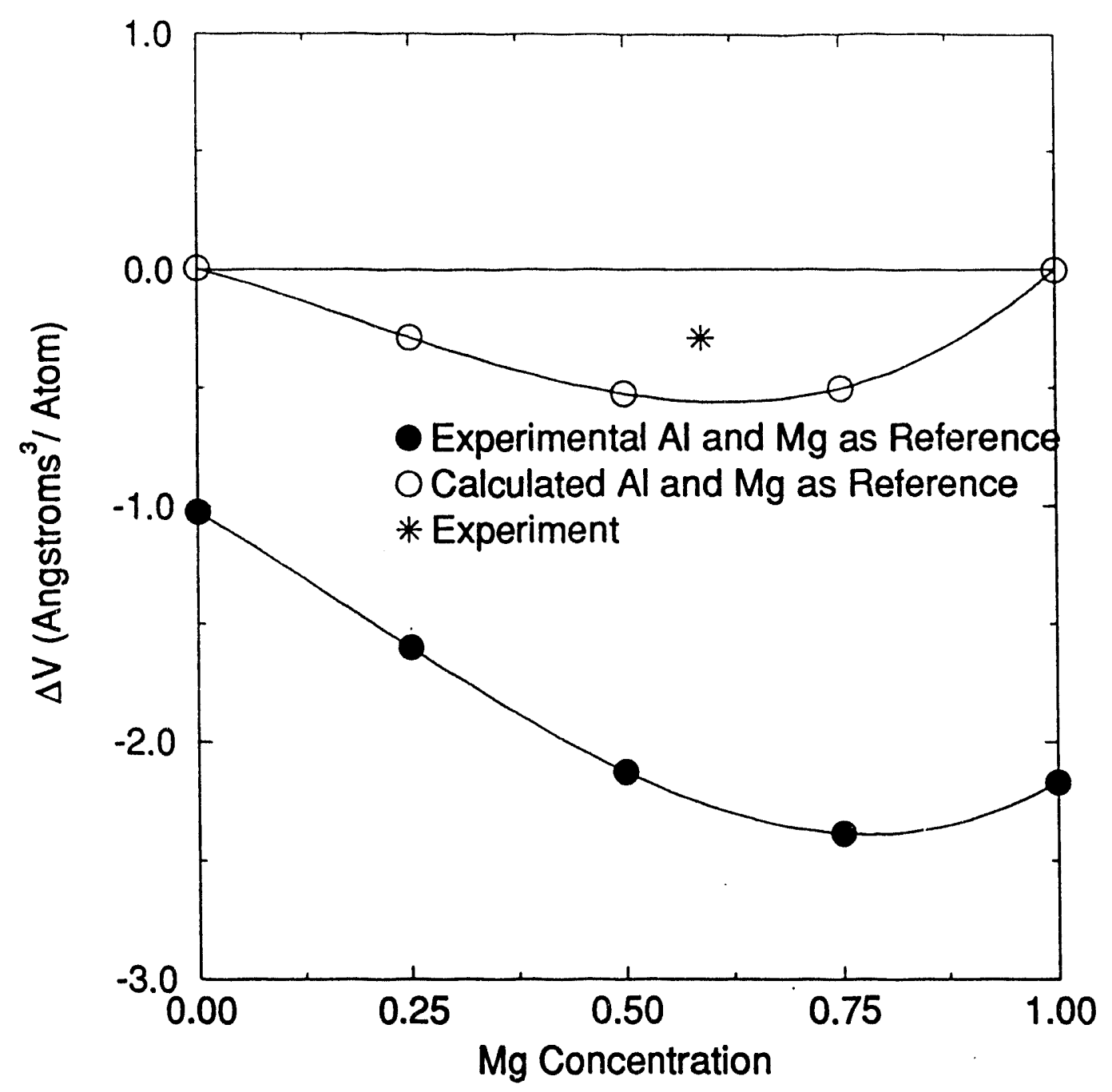

Figure 2: Change in Volume Per Atom for $A l_{1-x} M g_{x}$. Experiment is from Ref. 22.

requires the same number of operations, there is no problem with latency - that is some processors lying idle waiting for the results of others:

We have applied these algorithms to aluminum supercells of 32,108 , and 256 atoms. These are just cubic supercells with edge length $2 a, 3 a$, and $4 a$, respectively (aluminum is face centered cubic (fcc) so there are 4 atoms per cube of length $a$ ).

We first timed the algorithm for comparison on the Cray Y-MP C90, the Intel iPSC/860, and the IBM RS/6000 (Model 320). For the 108 atom supercell, there were approximately 200 states and 2000 plane waves (that is the $h$ matrix was $2000 \times 2000$ ). The C90 required 0.3 minutes per iteration, and the RS/6000 6.6 minutes. The $\mathrm{iPSC} / 860$, using 8 nodes in parallel required 2.8 minutes. Other things being equal, with 10 times as 


\section{Heats of Formation for Simple (fcc-like) AlMg Compounds}

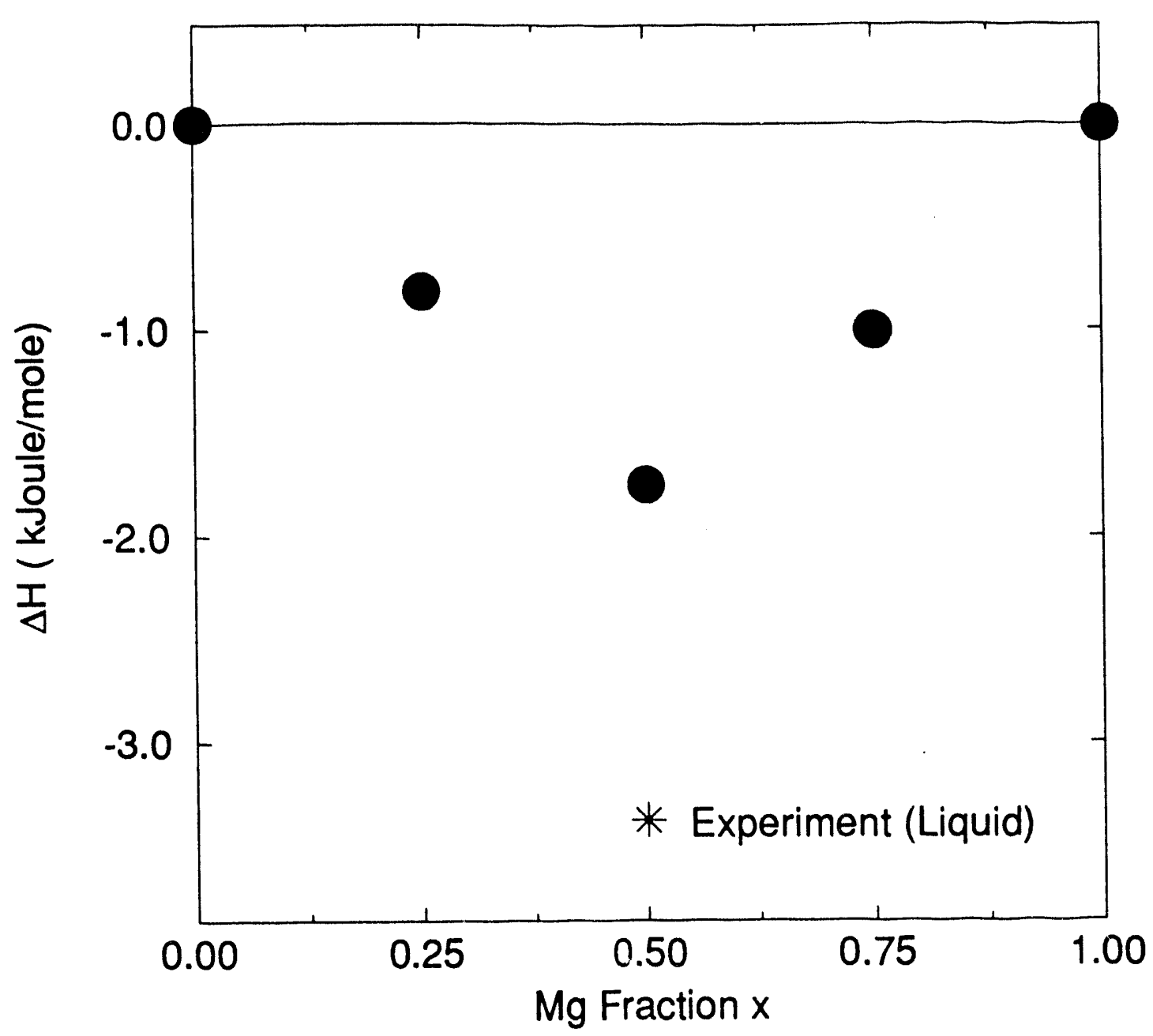

Figure 3: Heat of Formation For $A l_{1-x} M g_{x}$ for the $\mathrm{Ll}_{2}\left(\mathrm{Cu}_{3} \mathrm{Au}\right)$ and $\mathrm{Ll} l_{0}(\mathrm{Cu} \mathrm{AuI})$ Structures. Experiment is from Ref. 23.

many nodes, the computation time would about equal that of the Cray. Current parallel machines typically have 500-2000 nodes, so this kind of scale up is quite feasible. On the other hand, as the system size becomes larger the memory requirements and the time for the subspace diagonalization increase which will limit the size attained. We believe however that systems with $\sim 500$ atoms will be easily studied and this means that many interesting metallurgical structures are amenable to first principles calculations.

We have also calculated the unrelaxed vacancy formation energy. This was done by calculating the total energy of the defect free supercell, $E_{0}$, and the cell containing the vacancy $E_{v}$.

The vacancy formation energy is given by

$$
\Delta=E_{v}-\frac{X-1}{. V} E_{0}
$$


Table 2: Vacancy Formation Energy For $A l$

\begin{tabular}{|c|c|c|c|}
\hline \# Atoms & $1 k$ Point & $4 k$ Points & $10 k$ Points \\
\hline 32 & $-0.3 \mathrm{eV}$ & $0.61 \mathrm{eV}$ & $0.49 \mathrm{eV}$ \\
108 & $-0.5 \mathrm{eV}$ & & \\
256 & $1.2 \mathrm{eV}$ & & \\
& & & \\
\hline Expt & $(0.75 \pm 0.1) \mathrm{eV}$ & & \\
\hline
\end{tabular}

where $N$ is the number of atoms in the defect free cell. The results depend on the number of $k$ points used to sample the Brillouin zone of the supercell. The common practice for Car-Parrinello type simulations has been to choose a single $k$ point, usually $k=0$, also known as the $\Gamma$ point. From Table II, we see that for 32 and 108 atom supercells the vacancy formation energy actually has the wrong sign! For 256 atom supercells it provides an overestimate to the experimental value which is $0.7 \pm 0.1 \mathrm{eV}$ [21]. Using 4 or $10 k$ points in the Brillouin zone leads to.reasonable values with only 32 atom supercells. Further calculations to track down the effects of the $k$ point sampling and supercell size are in progress.

\section{Conclusion}

In conclusion, we have shown that the techniques pioneered by Car and Parrinello can be applied to metallic alloys where large numbers of atoms are common. These techniques also lend themselves readily to parallel computing architectures and further improvements in our computational abilities are expected soon.

\section{Acknowledgment}

This work was supported by the Division of Materials Sciences, Office of Basic Energy Sciences, U.S. Department of Energy under Contract No. DE-AC02-76CH00016, and by a grant of computer time at the National Energy Research Supercomputer Center, Livermore, California.

We would also like to thank R. M. Wentzcovitch, who kindly provided the pseudopotential program used for the $A l-M g$ alloys. 


\section{$\underline{\text { References }}$}

1. R. Car and M. Parrinello, "United Approach for Molecular Dynamics and Density Functional Theory," Physical Review Letters, 55 (1985), 2471-2474.

2. M.C. Payne, M.P. Teter, D.C. Allan, T.A. Arias, and J.D. Joannopoulos, "Iterative Minimization Techniques for $A b$ Initio Total-Energy Calculations: Molecular Dynamics," Reviews of Modern Physics, 64 (1992), 1045-1097.

3. M.F. Ashby and D.R.H. Jones, Engineering Materials 2 (Oxford, UK: Pergamon Press, 1986).

4. J.E. Hatch, ed., Aluminum, Properties and Physical Metallurgy (Metals Park, OH: American Society for Metals, 1984).

5. A.K. Vasudevan and R.D. Doherty, eds., Aluminum Alloys - Contemporary Research and Applications, Vol. 31 (San Diego, CA: Treatise on Materials Science and Technology, Academic Press, 1989).

6. T.B. Massalski, ed., Binary Alloy Phase Diagrams (Metals Park, OH: American Society for Metals, 1990).

7. W.E. Pickett, "Pseudopotential Methods in Condensed Matter Applications," Computer Physics Reports, 9 (1989), 115-197.

8. N. Troullier and J.L. Martins, "Efficient Pseudopotentials for Plane-Wave Calculations," Physical Review B, 43 (1991), 1993-2006.

9. A. Pasquarello, K. Laasonen, R. Car, C. Lee, and D. Vanderbilt, "Ab Initio Molecular Dynamics for d-Electron Systems: Liquid Copper at 1500K," Physical Review Letters, 69 (1992), 1982-1985.

10. L. Kleinman and D.M. Bylander, "Efficatious Form for Model Pseudopotentials," Physical Review Letters, 48 (1982), 1425-1428.

11. G.W. Fern ando, G.-X. Qian, M. Weinert, and J.W. Davenport, "First Pinciples Molecular Dynamics for Metals," Physical Review B, 40 (1989), 7985-7988.

12. J.L. Buchanan and P.R. Turner, Numerical Methods and Analysis (New York, NY: McGraw-Hill Inc., 1992), 597.

13. I. Stich, R. Car, and M. Parrinello, "Bonding and Disorder in Liquid Silicon," Physical Review Letters, 63 (1989), 2240-2243.

14. G.-X. Qian, M. Weinert, G.W. Fernando, and J.W. Davenport, "First Principles Calculation of the Activation Energy for Diffusion in Liquid Sodium," Physical Review Letters, 64 (1990), 1146-1149. 
15. G.-X. Qian and J.W. Davenport, "First Principles Molecular Dynamics Study of Liquid Aluminum," (ui.published).

16. J. Pasciak et al., (unpublished).

17. G.B. Bachelet, D.R. Hamann, and M. Schluter, "Pseudopotentials That Work: From H to Pu," Physical Review B, 26 (1982), 4199-4228.

18. P.K. Lam and M.L. Cohen, "Ab Initio Calculation of the Static Structural Properties of Al," Physical Review B, 24 (1981), 4224-4229.

19. M.Y. Chou and M.L. Cohen, " $A b$ Initio Study of the Structural Properties of Magnesium," Solid State Commun., 57 (1986), 785-788.

20. R.M. Wentzcovitch and M.L. Cohen, "Theoretical Model for the hcp-bcc Transition in Mg," Physical Review B, 37 (1988), 5571-5576.

21. R.W. Cahn and P. Haasen, eds., Physical Metallurgy (Amsterdam: North Holland Physics Publishing, 1983), 1146.

22. Pearson's Handbook of Crystallographic Data for Intermetallic Phases, 2nd Edition (Metals Park, OH: American Society for Metals, 1991).

23. R. Hultgren, P.D. Desai, D.T. Hawkins, M. Gleiser, and K.K. Kelley, Selected Values of the Thermodynamic Properties of Binary Alloys (Metals Park, OH: American Society for Metals, 1973). 

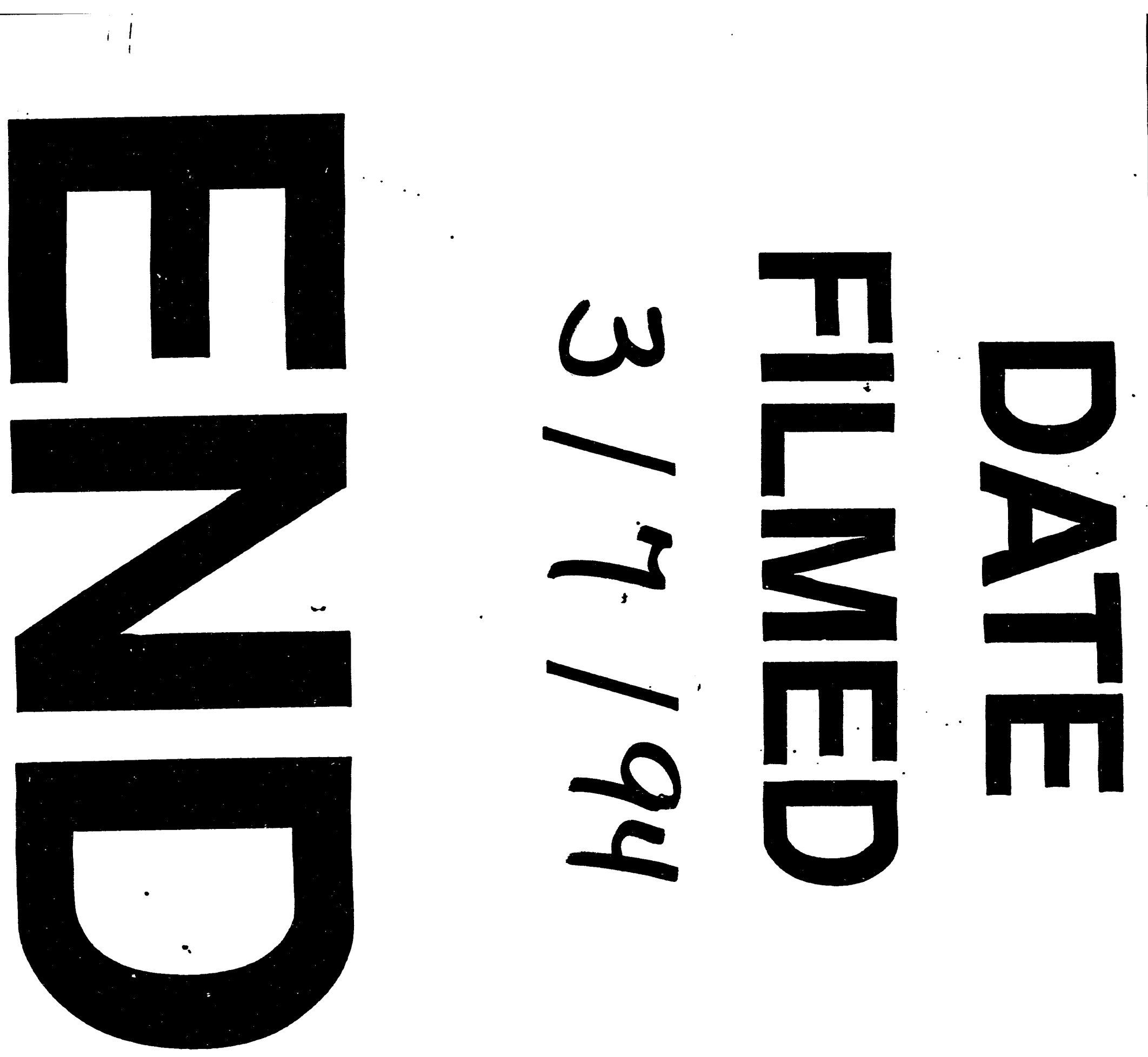